Recibido: junio de 2015

Aprobado: septiembre de 2015

DOI:

http://dx.doi.org//0.15332/rev.m.v/2il.1926

\section{CENTRO HISTÓRICO Y ARQUITECTURA CONTEMPORÁNEA: APROXIMACIÓN TEÓRICA PARA EL SIGLO XXI*}

\author{
William Pasuy Arciniegas** - Universidad de la Salle, Colombia
}

Centro Cultural "Gabriel García Márquez", Fondo de Cultura Económica y Catedral Primada de Bogotá, Centro Histórico de Bogotá (Colombia).

Fuente: William Pasuy Arciniegas, 2015

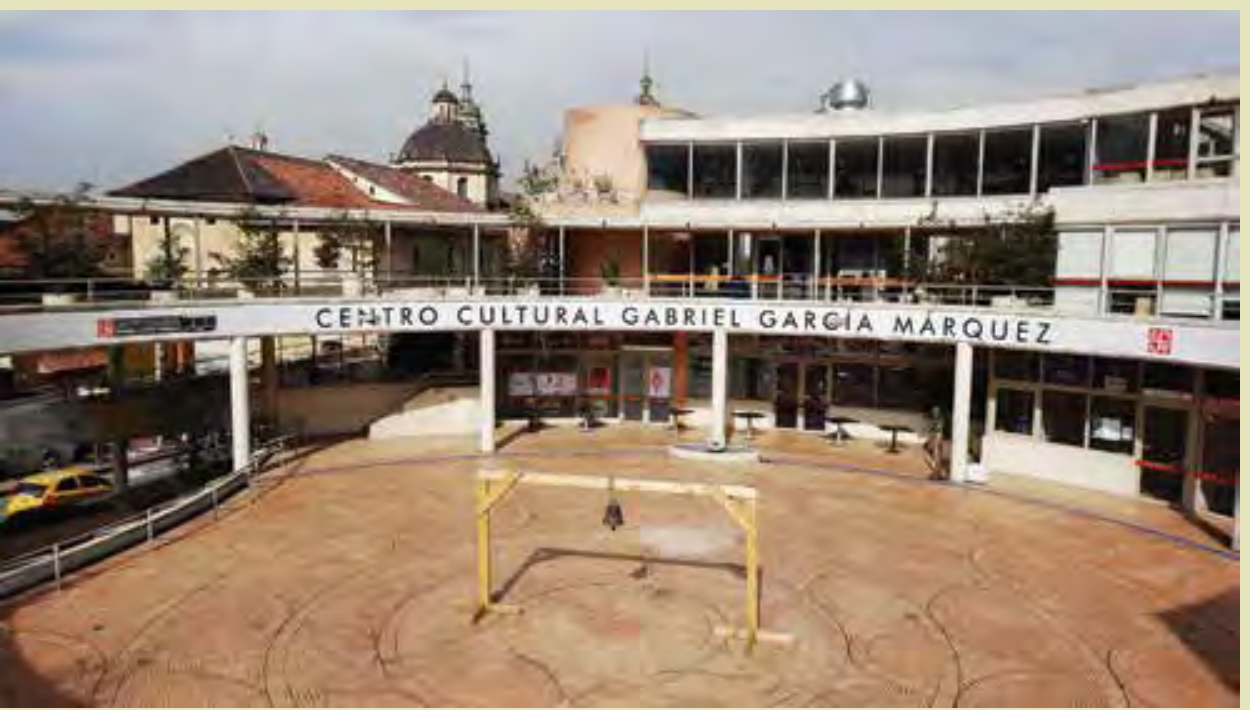

\section{RESUMEN}

La generación de arquitectura contemporánea en centros históricos, como contexto patrimonial, ha sido un proceso permanente durante siglos, revelando la evolución del pensamiento, las transformaciones socioculturales y los momentos históricos de los territorios y sus comunidades; sin duda, estos procesos han dejado huellas físico-espaciales representadas en morfologías urbanas y lenguajes arquitectónicos, cuyas lecturas por períodos permiten identificar, caracterizar y valorar las continuas, evolutivas y actuales producciones arquitectónicas.

Hoy, la generación de arquitectura contemporánea en centros históricos tiene vital responsabilidad: conservación del patrimonio como proceso evolutivo, contextualización de nuevas producciones espaciales, articulación y/o diferenciación entre lo antiguo y lo actual, transformaciones sociales, participación y apropiación social del patrimonio, entre otras; es una estrategia alterna a clásicas intervenciones de restauración aislada de edificaciones o contextos con valor patrimonial.

En consecuencia, con el presente pretexto teórico no se pretende definir parámetros o respuestas que permitan proyectar la inserción de arquitectura contemporánea en centros históricos, sino indagar sobre conceptos entre arquitectura contemporánea y centro histórico, con el ánimo de generar reflexiones.

\section{PALABRAS CLAVE}

Centro histórico, patrimonio, arquitectura contemporánea, articulación. 


\section{HISTORICAL CENTER AND CONTEMPORARY ARCHITECTURE: A THEORETICAL APPROACH FOR THE XXI CENTURY}

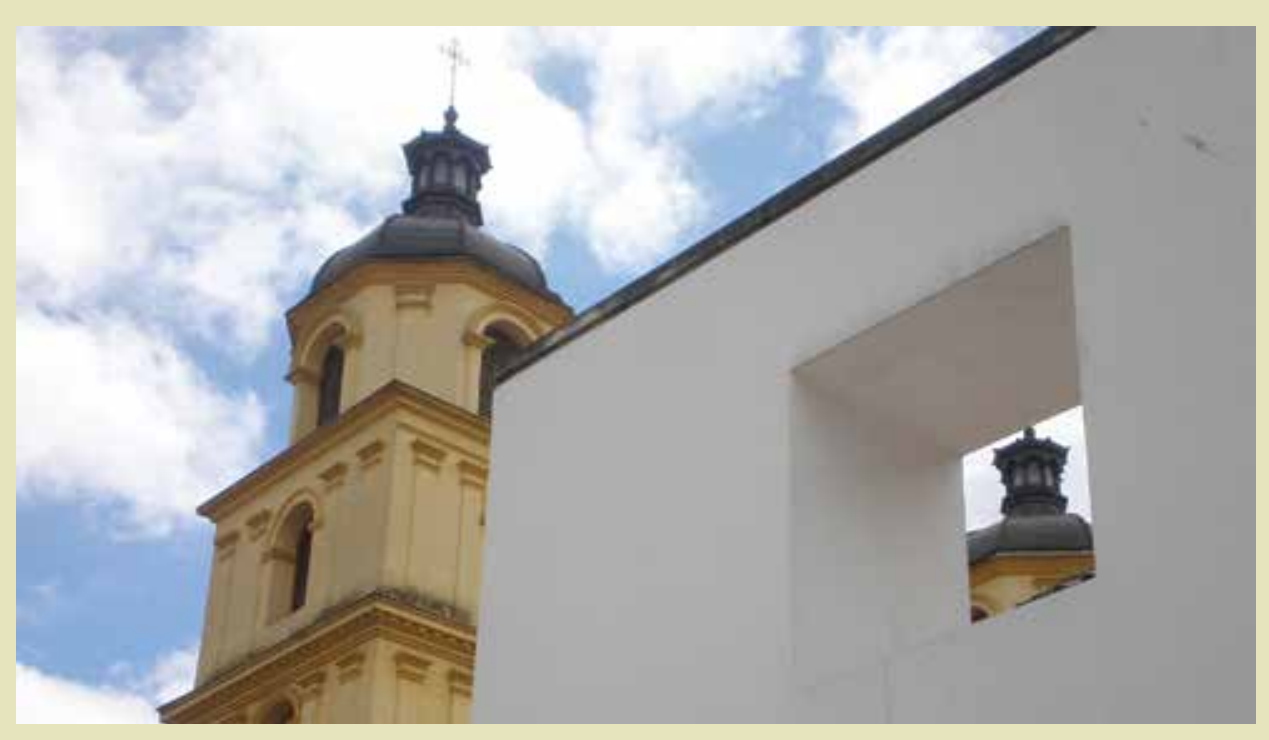

Detalle Museo de Arte del Banco de la República y Templo de La Candelaria, Centro Histórico de Bogotá (Colombia). Fuente: William Pasuy Arciniegas, 2015.

\begin{abstract}
Contemporary architecture in historic centres and heritage contexts, and its ongoing process of development over the centuries, nowadays reveal the evolution of thought, social and cultural transformations, and historic events of different territories and their communities. Certainly, these processes have left physical and spatial marks imprinted in the urban environment in the form of urban morphologies and architecture languages, which are traceable through readings of different historic periods. This approach permits to identify, characterize and value continuous, evolutionary and current architectural productions.
\end{abstract}

Nowadays, contemporary architecture in historic districts faces relevant challenges and entails important responsibilities: the evolutionary process of heritage conservation, contextualization of new spatial developments, articulation and / or differentiation of new and ancient architectural styles, social participation and heritage appropriation among others, as alternative strategies to classic approaches that treat restoration of patrimonial buildings and heritage contexts as isolated elements.

Consequently, the following article attempts to inquire about concepts extracted from contemporary architecture and historic centres with the aim of generating debate. However, this theoretical framework does not intend to define specific parameters for the insertion of contemporary architecture in historic districts.

\section{KEYWORDS}

Historic centre, heritage, contemporary architecture, articulation. 


\section{INTRODUCCIÓN}

El tema planteado surge por la inquietud de reflexionar sobre la relación existente entre centro histórico y arquitectura contemporánea a partir de sus conceptos; investigación que actualmente se adelanta como tesis doctoral en el Programa Interinstitucional de Doctorado en Arquitectura - Línea Patrimonio, en la Universidad Michoacana de San Nicolás de Hidalgo en Morelia - México.

En centros históricos, la generación de arquitectura contemporánea, que en muchos casos se la asimila a "progreso", ha sido un proceso evolutivo como consecuencia de acontecimientos socioculturales y transformaciones espaciales por periodos que generan nuevas espacialidades en contextos rurales o urbanos que poseen características patrimoniales; los ejercicios proyectuales tienen la posibilidad de otorgar renovados sellos o huellas del momento en cuanto al pensamiento y cotidianidad que los pobladores tenían en eras específicas y se pueden reconocer a través de la arquitectura y sus contextos de origen. Estas acciones no solo permiten identificar y valorar el patrimonio o impulsar su conservación, sino caracterizar la arquitectura contemporánea que se integra en dichos contextos como parte integral de un proceso histórico, donde es posible adelantar su lectura y reflexión crítica desde el momento mismo de su creación, como hecho coyuntural y puesta al servicio de las comunidades, sin la necesidad de esperar décadas para adelantar posibles evaluaciones críticas.

El presente ejercicio de reflexión tiene como alcance aproximar a un marco conceptual que permita consolidar aportes sobre arquitectura contemporánea en centros históricos, reiterando que, por el momento, los aspectos de temporalidad y espacialidad no son una camisa de fuerza; esta reflexión bien podría ser insumo para posteriores estudios de caso.

\section{LA FUNDAMENTACIÓN CONCEPTUAL PRELIMINAR: LO PATRIMONIAL Y LO CONTEMPORÁNEO}

Existen dos conceptos de pertinente abordaje para la reflexión: lo patrimonial y lo contemporáneo (como acto cultural); abordados de manera aislada constituyen, en primer momento, el fenómeno investigativo de integración y articulación entre el pasado, presente y futuro.

Lo patrimonial, desde lo conceptual y la praxis, puede referir a los recursos naturales, bienes y manifestaciones culturales que poseen valores particulares o excepcionales; donde la trilogía entre memorias, territorios y comunidades han otorgado su caracterización. Por citar casos: en reservas o parques naturales, centros históricos, edificaciones, objetos, tradiciones o saberes colectivos.

Según el Diccionario de la Real Academia Española (1992), la palabra "patrimonio" proviene del latín "patrimonium" y se define como "hacienda que alguien ha heredado de sus ascendientes"; por su parte, para la UNESCO (s.f.) el concepto ha ido evolucionando con el tiempo e identifica dos naturalezas, material e inmaterial, ya que el patrimonio no está referido exclusivamente a los objetos, artefactos o monumentos producidos por el hombre, sino que va más allá e involucra las tradiciones y expresiones que hemos heredado de nuestros antecesores y que se siguen transmitiendo, por ejemplo las tradiciones orales, fiestas y técnicas artesanales.

De igual forma, en Colombia se encuentra la legislación cultural, emitida por el Congreso de la República, la cual define el patrimonio cultural desde los estamentos legales, espe- 
cíficamente en la ley I I 85 de 2008 que modifica la ley 397 de 1997 correspondiente a la Ley General de Cultura. En el artículo cuarto de la mencionada ley I 185, referido a la “integración del patrimonio cultural de la Nación”, se precisa el patrimonio cultural como "todos los bienes materiales, las manifestaciones inmateriales, los productos y las representaciones de la cultura que son expresión de la nacionalidad colombiana". Dentro de esta definición entran las tradiciones y evidencias culturales contenidas en diferentes materiales y soportes, lenguas, dialectos de los grupos nativos, bienes inmuebles, entre otros.

Por su parte, el concepto de lo contemporáneo se ha comprendido en concordancia a las realidades evolutivas e intermitencias que articula el pasado y el futuro, sin cronología determinada; refiere al resultado actual del pensamiento humano acorde a su momento histórico, donde se revelan sus creaciones a partir de las necesidades socioculturales y físico-espaciales de una comunidad en su territorio, desde sus realidades o contextos específicos, a manera de rótula.

El Diccionario de la Real Academia Española (1992) acota que la denominación de "contemporáneo" proviene del latín "contemporaněus" y lo define como: "existente en el mismo tiempo que otra persona o cosa / Perteneciente o relativo al tiempo o época en que se vive". Por su parte los autores José Gandarilla (Doctor en Filosofía), Ramón Ramos (Catedrático de Sociología) y Guadalupe Valencia (Investigadora en Ciencias y Humanidades), en su libro "Contemporaneidad(es)" (2012), consolidan, desde diversos puntos de vista disciplinar y de autores, el concepto de lo contemporáneo. Por ejemplo, Guadalupe Valencia puntualiza: "Contemporáneos se supone que son los individuos, los grupos y las sociedades que, por el sólo hecho de estar vivos, pueden sentirse co-partícipes de sus propios mundos, herederos de mundos sociales preexistentes, precursores de tiempos por venir [...]"; ahora, Juan Carlos Huidrobo (Psicólogo y Sociólogo) invita a reflexionar por lo contemporáneo:

[...] tiene implicaciones importantes en el desarrollo y conceptualización de las sociedades. Tal envuelve no únicamente cómo se vive la dimensión temporal... sino de igual manera cómo es que el mundo temporal es observado y reflexionado conceptualmente... si la función de cada presente, como actualidad, es generar tiempo, forjar un nuevo pasado y futuro, la pregunta, hoy, por lo contemporáneo es, en estricto sentido, el inicio de una, nueva, historia reflexiva. (Gandarilla, Ramos y Valencia, 2012, p. 139.)

Finalmente, Emma León (Investigadora de temas multidisciplinarios) aborda el tema de la contemporaneidad como:

[...] una designación de pertenencia colectiva y no una modalidad particular del tiempo, tal y como llega a suceder con los términos presente, pasado o futuro [...] la temporalidad de lo contemporáneo es de una entidad humana con capacidad para adherirse a ella colectivamente $y$, con base en eso, fijar un criterio de pertenencia -por tanto de exclusión y diferencia- respecto al rango de realidad en que se ubica, sea en relación a las de su misma clase o respecto a otras. (Gandarilla et al, 20I2, p. I3).

Entonces, como reflexión inicial, lo patrimonial y lo contemporáneo transitan en tiempo y espacio porque se contienen, interactúan y articulan simultáneamente, donde las preexistencias o el denominado "pasado" es punto de partida de lo actual en colectividad, es decir, lo contemporáneo surge desde la experiencia y es puente de un futuro por experimentar. 


\section{DIÁLOGO ENTRE EL CENTRO HISTÓRICO Y ARQUITECTURA CONTEMPORÁNEA}

En un segundo momento, el fenómeno investigativo se aproxima a los diálogos entre el centro histórico y su devenir transicional (lo que vendrá) con la arquitectura contemporánea. Para ello, nuevamente es importante aproximarse a los conceptos sobre centro histórico y arquitectura contemporánea.

El centro histórico, identificado con diversas denominaciones en diferentes latitudes del mundo como centro fundacional, sector antiguo, antigua ciudad, área céntrica o conjunto histórico, puede ser subjetivo si se desarticula de sus contenidos naturales y culturales materiales e inmateriales. Es decir, este debe referir tanto a la evolución urbana y creaciones arquitectónicas en un área específica por períodos, como a tradiciones, acontecimientos y memorias que sus pobladores le han impregnado en el tiempo (historia) a sus creaciones espaciales regenerativas; desde sus contextos socioculturales a sus geografías, propendiendo por su lectura y entendimiento objetivo, en tanto que el centro histórico lo hace la colectividad con sentido social y procesos absolutamente dinámicos.

La concepción y denominación de centro histórico surge a nivel global en la primera mitad del siglo $X X$, donde se reconoce en las ciudades áreas antiguas, generalmente fundacionales y sectores con características históricas, geográficas y sociales que poseen jerarquía y centralidad. Asimismo, se inicia una política pública y normativa sobre dichas áreas particulares. Al respecto, Fernando Carrión (citado en Martínez, 2004) afirma:

La definición empírica de un centro histórico es un acto de política urbana, que implica una acción de un sujeto patrimonial con voluntad consciente. El problema radica en determinar, desde qué conceptos y con qué metodología se lo hace: si es bajo los atributos urbanos o arquitectónicos -lo tradicional-, o bajo las relaciones que le convierte en un eje -lo nuevo. (p. 37)

Para el caso de Colombia, el origen de la denominación se remonta a 1959 y la Ley 163 , reglamentado mediante Decreto 264 de 1963 donde se refiere a "sectores antiguos", incluyendo a Bogotá, como sectores que son integrados por "calles, plazas, plazoletas, murallas y demás inmuebles originarios de los siglos XVI, XVII, XVIII y principios del XIX" (Artículo $4^{\circ}$-Parágrafo). De igual forma, se asimila la denominación de centro histórico para el caso de Bogotá mediante Decreto 678 de 1994. El documento CONPES 3658 (2010) aborda el concepto de centro histórico como:

[...] oportunidades de desarrollo para un grupo de municipios del país que por sus características patrimoniales particulares representan un potencial excepcional para la dinamización de ciudades y municipios, a través de la oferta de espacio público, turismo cultural, vivienda y servicios de calidad. (p. 13)

Ahora bien, los centros históricos poseen una cualidad evolutiva en doble dirección: desde la físico-espacial, como un área que sintetiza las espacialidades de diferentes períodos; y desde lo sociocultural, por la caracterización que le otorgan colectivamente sus pobladores a la espacialidad (de generación en generación) como resultado de la relación de diversos contextos políticos, económicos, sociales, religiosos, tradiciones, etc. Valdría la pena iniciar las reflexiones sobre las producciones espaciales en dichas áreas como constructos sociales periódicos, en otras palabras, cada momento histórico generó progresivamente contemporaneidades como hitos de tiempo y espacio. De esta manera, lo contemporáneo 
efectivamente es la actualidad en cada temporalidad y espacialidad reconocida a través de diferentes productos, entre ellos, la arquitectura contemporánea. Por ello, no es absurdo afirmar que existió y existe contemporaneidad en la Colonia, la República, la Modernidad e incluso en la actualidad.

Con todo, compete enfocarse en el presente actual (así, redundante), y referirse a la arquitectura contemporánea, aquella que se proyecta para visibilizar las realidades y necesidades, a buscar (se quiera o no) presencia o articulación contextual, formal, funcional y material, donde ya hay contexto, forma, función y materia (círculo vicioso y recíproco). Esto con el fin de servir como puente entre un pasado con valores y nuevas expresiones prospectivas, destacando las actuales dinámicas físico-espaciales y socioculturales.

La arquitectura contemporánea generada en contextos antiguos, históricos patrimoniales es un proceso proyectual subjetivo y actual de evolución, sin delimitación de tiempo específico, sin espacialidad definida, dinámica y sin "sello" histórico denominado; desde la relatividad, es una producción material e inmaterial que conjuga las actuales dinámicas espaciales y culturales para ser parte del entorno, que aspira relacionar armónicamente contextos, formas, funciones y materialidad de manera sistémica para beneficio colectivo; un presente coyuntural entre lo preexistente y el porvenir.

Luís Madia en su libro "Introducción a la arquitectura contemporánea" (2003) plantea tres aspectos reflexivos:

[...] La necesidad de comprender a la Arquitectura en su vínculo permanente con la Cultura de la que es una parte plenamente involucrada y reflejante. Despertar y ampliar el interés por quienes esto lean, de la necesidad de entender a la Arquitectura como testigo palpable de la evolución social en el mundo y en lo regional, nacional y local, propendiendo a su valoración testimonial y su preservación. Y finalmente buscar que su discurso crítico tenga acogida por afuera de la Disciplina específica para amalgamarse en un diálogo más fructífero con los usuarios de Arquitectura, que somos todos. (p. 9)

Pero no todo apunta a un buen panorama. La inserción de arquitectura contemporánea también está constituyendo afectaciones negativas por diferentes factores, como el turismo cultural, las presiones económicas, la ausencia de políticas públicas, la llegada de nuevos pobladores, entre otros, que quizás generan nuevas dinámicas y por ende, nueva arquitectura sin atención a contextos y evolución histórica, pese a sus continuidades y discontinuidades. Carlos Flores Marini (1937-20I5), en uno de sus últimos textos consignados en uno de los capítulos del libro "Nuevos Paradigmas: Carta de Venecia 50 años" de los editores López y Vidargas, alude a una reflexión sobre las posibles y preocupantes transformaciones de los centros históricos por agentes exógenos a las condiciones del lugar o a los pobladores raizales que generan la arquitectura del momento:

[...] Ahora un nuevo "paradigma" acecha a los enunciados de la Carta de Venecia. La arquitectura de los migrantes ha hecho su aparición en cientos de poblados, ciudades medias y aún grandes. Aunque en estas últimas por su dimensión cuentan menos y no inciden, esperemos, en sus centros históricos los millones de trasterrados que con sus remesas alientan la construcción de diferentes modelos de hábitat, casi siempre estrambóticos. Entre ellos se juntó lo exótico con lo terrenal... la nueva arquitectura de los migrantes se va a constituir en edificios simbólicos sin duda alguna, y con Chamanes y 
Marías Sabinas podemos hacer casas mágicas. Con todo lo grotesco que nos parezca, esto es una realidad que tendrá que ser abordada por las autoridades municipales en sus reglamentos de imagen urbana. (Flores, 2014, p. 97)

La relación entre arquitectura contemporánea y contextos con patrimonio merecen atención a través de una previa revisión de la historia y de la cotidianidad que proyecte procesos dimensionales de conservación y salvaguardia, interacción entre lo material y lo inmaterial, acento de espacio y tiempo, creación y proyección; Ibo Bonilla Oconitrillo convoca en su reflexión en torno a la Carta de Oaxaca 2008: "[...] a los profesionales relacionados a que cada inmueble, cada sitio y cada centro histórico que sea intervenido, se haga mediante estrategias y técnicas claramente orientadas a la sustentabilidad, como soporte de su supervivencia" (Citado en AECID, 20I I, p. 2I).

Con respecto a la reflexión de continuidad, la arquitectura contemporánea como definición y concepto, quizás se está convirtiendo en un "cliché" o una mal denominada "tendencia o estilo" arquitectónico desde la temporalidad en que vivimos, que es nuestra realidad. Bien se puede hacer una crítica reflexiva sobre su integración en contextos patrimoniales $y$, en especial, en los centros históricos, logrando una arquitectura de integración que, con el paso del tiempo, logrará su denominación histórica a partir de sus preexistencias. También es cierto que la tan anhelada "integración" puede ser subjetiva junto con los procesos proyectuales hipotéticos desconocidos, antes de la utilización y apropiación de la arquitectura por sus usuarios; solo la interacción presente entre contextos, formas, función y materialidad reflejan la cotidianidad, lo social, apreciación y valoración (o no) de la arquitectura contemporánea en los centros históricos.

\section{EL PRESENTE: PUENTE ENTRE PASADO Y FUTURO. ARQUITECTURA CONTEMPORÁNEA Y CENTRO HISTÓRICO ¿PROCESOS DE AFINIDAD O DE CONTRASTE?}

Un tercer momento, la relación entre las preexistencias y lo que vendrá, como continuidad al fenómeno de investigación. Diversas formas de establecer conceptos en un mismo camino: arquitectura contemporánea en centros históricos, integración de arquitectura contemporánea en ciudades patrimoniales, arquitectura contemporánea al rescate del patrimonio cultural, intervenciones contemporáneas en la ciudad histórica, en fin, un vasto número de denominaciones que revelan una creciente preocupación por el tema.

Fernando González Gortázar (2014) hace una interesante reflexión sobre el pasado y el futuro frente a la producción actual:

En la cultura todo vuelve y se reinventa, y es al mismo tiempo nuevo y antiquísimo; es el tiempo de circular en el que todo gira [...] El tiempo de la cultura, y por tanto de la arquitectura, es como una hélice o helicoide en el espacio, que vista desde cierto ángulo parece que regresa al mismo sitio, pero desde otro se ve que siempre se está prolongando y moviendo hacia adelante... es interesante el asunto de la arquitectura que se construye en contextos urbanos antiguos o característicos. Ante esto siempre existe la tentación, sobre todo, por parte de ciertas autoridades, a imitar, de reproducir los estilos del pasado; y por parte de los arquitectos, frecuentemente existe la tentación contraria: la de hacer una declaración extrema de que nuestros tiempos son distintos. (p 160). 
Los puntos de vista podrían ser extremos y peligrosos, desde la reproducción de estilos que pretenden traer un pasado al presente, o la generación de arquitectura actual que rompa con la evolución del lugar sin argumentación ni articulación. El proceso debería propender por la generación de respuestas arquitectónicas a manera de pausas reflexivas (al intentar entender las etapas evolutivas que se traslapan entre sí para la creación), a la articulación de la arquitectura por afinidad o por contraste, siempre en la búsqueda compleja de la integración y la relación con sus contextos multidimensionales, como son: lo sociocultural (inmaterial) y lo físico-espacial (material).

Si el fin es la articulación entre lo vivido y el porvenir a través de la creación arquitectónica, cualquier alternativa podría ser viable, pero estas podrían redundar en dos grandes grupos: la arquitectura de integración por afinidad o por contraste; en ambos casos, valorando los procesos históricos, sociales, naturales y culturales como preexistencias objetivas.

Por afinidad, se debería propender por las relaciones armónicas continuas entre sus contextos, formas, funciones y materialidades pasadas con la nueva producción proyectual, donde no solo se tenga en cuenta recurrentes aspectos que apuntan al cumplimiento de normativas de homogeneidad (como el manejo de alturas, paramento, proporción y escala, materiales y lenguajes), sino también usos, actividades, oficios y tradiciones. Lo anterior, si las condiciones proyectuales y necesidades del proyecto así lo permiten; entendiendo esto como un proceso pertinente de correlación, cuyo resultado no genere impactos de protagonismo o arquitecturas de autor o corporativas, sino de correspondencia evolutiva que permita la lectura de pensamiento y necesidades del momento histórico actual. Es decir, una creación más pasiva y quizás hasta neutral.

En cambio, si es por contraste, las relaciones armónicas pueden ser aparentemente discontinuas y jerárquicas, evidentes y trasformadoras de contextos, formas, funciones $y$ materialidades. De aquí, que, a pesar de dar cumplimiento a normativas, bien se podría generar creaciones heterogéneas a través de discontinuidad de alturas, paramentos $y$ materiales en la búsqueda de innovadores lenguajes, alterando, sin afectaciones negativas sino como complementos, los usos y actividades preexistentes. También, lo planteado conlleva a la proyección de correspondencias en la evolución de lugar y pensamiento, así mismo, evidencia el actual momento histórico con un acento de creación activo donde la neutralidad no sería su característica.

Cualquier alternativa a desarrollar, por afinidad o por contraste, se deben caracterizar por un concepto ineludible: la calidad e integralidad de la creación, que, sin duda, podrá caracterizar el momento actual y puente entre pasado y futuro. En últimas, la articulación es sinónimo de coyuntura temporal, espacial y de memoria colectiva. Al respecto, se fundamenta un anhelado resultado, independientemente al proceso, en lo consignado en el siguiente texto:

Ésa (sic) es la clave: la calidad. No debemos falsear el momento histórico, pero tampoco agredir al contexto urbano. No hay que olvidar que lo antiguo y lo moderno, si tienen calidad, conviven perfectamente, mientras que lo auténtico o lo ficticio chocan siempre. De tal manera que una actitud de modestia, de decir la verdad, de respeto y de calidad, es el único acercamiento posible para hacer arquitectura actual en contextos históricos... abraca a los edificios, los árboles, el pavimento de las calles, el mobiliario urbano: si alteras uno, alteras todo. También abarca el patrimonio intangible, las tradiciones, las maneras de ser y de vivir (es decir, las formas de usar la ciudad), la música que se escucha, el nombre de las poblaciones y las calles... Puede haber ocasiones en las que la 
protección de uno de esos intangibles sea determinante para acciones urbanas

(González, 2014, p I6I).

\section{CONCLUSIÓN}

Como se acotó al inicio, aproximarse a los conceptos es aportar a la reflexión en torno a la generación de arquitectura contemporánea en centros históricos, y por ahora, ampliar la discusión a casos de estudio sería precipitado, ya que la fundamentación, conocimiento y reconocimiento de conceptos son el punto de partida para el análisis y la producción intelectual. Sin embargo, a manera de reflexión y cuestionamiento, se plantean las siguientes cavilaciones que no pretenden de ninguna manera cerrar el tema, sino, por el contrario, abrir brechas de continuidad frente a un tema cíclico que hoy, en muchos contextos, no se están visibilizando como alternativa de conservación y salvaguardia del patrimonio cultural.

Entonces, a manera de cierre y continuidad de pensamiento, pensar en la generación de arquitectura contemporánea en centros histórico debería invitar a las siguientes reflexiones:

- La arquitectura contemporánea en centros históricos no refiere a un nuevo proceso, por el contrario, es un devenir en permanente movimiento, suma de contemporaneidades que se presentan en cada momento histórico acorde a los contextos que generaron su producción proyectual, desde lo material e inmaterial.

- Sin duda, lo patrimonial y lo contemporáneo siempre se contienen a partir de sus particularidades de tiempo, espacio y memoria colectiva; están en permanente correlación, interacción y articulación simultánea, lo cual genera identidad y diferencia en los territorios y las comunidades que lo producen.

- La arquitectura contemporánea no puede ser vista, creada y analizada como un movimiento, tendencia y menos como un estilo arquitectónico; es una realidad presente que vivimos plural y colectivamente como constructo social; es una arquitectura de integración que con el paso del tiempo logrará su denominación histórica a partir de sus vínculos con las preexistencias.

- ¿Vale la pena restringir los procesos proyectuales a solo tipologías preexistentes, morfología típicas o materialidades en los centros históricos? Acaso, ino se estaría limitando la naturaleza de la creatividad en los procesos proyectuales, la cotidianidad y la colectividad histórica?

- La calidad de procesos proyectuales y resultado de la generación de arquitectura contemporánea en centros históricos, junto con aspectos materiales e inmateriales (por afinidad o por contraste), deben ser el fundamento de la integralidad espacio, tiempo y memoria, puente entre pasado y futuro que logre responsablemente articularse sistémica y multidimensionalmente a sus contextos, cotidianidad y colectividad de los pobladores. Por tanto, las arquitecturas contemporáneas en los centros históricos deben ser ineludiblemente de calidad y no de imitación. 
- La generación de arquitectura contemporánea en centros históricos debería tener un acento y enfoque a la conservación del patrimonio cultural de manera amplia y participativa, como estrategia no solo de su apropiación social, sino de conservación y salvaguardia del patrimonio cultural pertinente al momento histórico actual.

\section{REFERENCIAS}

Agencia Española de Cooperación Internacional para el Desarrollo (AECID). (Noviembre 7 al 10, 20I I). VIII Encuentro Gestión de Centros Históricos, Antigua, Guatemala.

Alcaldía mayor de Santa Fe de Bogotá. Decreto 678. Bogotá, 1994. Recuperado de http:// www.sdp.gov.co/portal/page/portal/PortalSDP/OrdenamientoTerritorial/Patrimonio/MarcoNormativo/DECRETO\%20678\%20DE\%201994.pdf

Congreso de Colombia. Ley I I85. Bogotá, 2008. Recuperado de http://www.icanh.gov. $\mathrm{co} /$ ?idcategoria $=209$ I

Consejo económico de política económica y social-CONPES. (2010). Lineamiento de políticas para la recuperación de los centros históricos de Colombia: documento CONPES 3658. Bogotá: Departamento Nacional de Planeación.

Flores, C. (2014). Reflexiones a 50 años de la carta de Venecia. En López, F, Vidargas, F. (Ed.), Los nuevos paradigmas de la conservación del patrimonio cultural: 50 años de la Carta de Venecia (9I-99). México, D.F.: Instituto Nacional de Antropología e Historia.

Gandarilla, J. Ramos, R. Valencia, G. (20I2). Contemporaneidad(es). Madrid: Ediciones Sequitur.

González, F. (2014). Arquitectura: pensamiento y creación. México: Editorial Universidad Nacional Autónoma de México.

Madia, L. (2003). Introducción a la arquitectura contemporánea. Buenos Aires: Editorial Nobuko.

Martínez, M. (2004). El centro histórico: objeto de estudio e intervención. Bogotá: Editorial Pontificia Universidad Javeriana.

Presidencia de la República de Colombia. Decreto 264. Bogotá, 1963. Recuperado de http://www.alcaldiabogota.gov.co/sisjur/normas/Normal.jsp?i= 1307

Real Academia Española. (1992). Diccionario de la Lengua Española, (I). Madrid: Instituto Cervantes.

UNESCO. (s.f.). ¿Qué es el patrimonio cultural inmaterial? Sin referencia de lugar y editorial. Recuperado de https://ich.unesco.org/es/que-es-el-patrimonio-inmaterial-00003 